\title{
The Variable Appearance of Third Ventricular Colloid Cysts: Correlation with Histopathology and the Risk of Obstructive Ventriculomegaly
}

\author{
(D) S.D. Khanpara, (D)A.L. Day, (D) M.B. Bhattacharjee, (D)R.F. Riascos, (D).P. Fernelius, and (D).D. Westmark
}

\begin{abstract}
BACKGROUND AND PURPOSE: While third ventricular colloid cysts may present as an incidental finding, they also harbor the potential to cause ventricular obstruction and sudden death. Herein we analyze the relationship between imaging appearance and the risk of obstructive ventriculomegaly.
\end{abstract}

MATERIALS AND METHODS: This is a retrospective review of the MR imaging appearance of 64 patients with colloid cysts, 46 of whom also had a CT scan, obtained by a tertiary hospital imaging report data base search over a 10-year period. Cysts were categorized by appearance on T2-FLAIR and correlated with patient age, cyst size, and the risk of obstructive ventriculomegaly. Histopathologic correlation was available for 28 cases.

RESULTS: The 64-patient cohort was 52\% female, median age 50 years (range 10 to 99 years). Cysts hyperintense on T2-FLAIR (53.1\%) were larger $(P<.001)$, occurred in younger patients $(P=.01)$, and had a higher risk of obstructive ventriculomegaly than homogeneously hypointense cysts (relative risk 6.18, 95\% Cl [2.04, 18.67]). Three patterns of T2 hyperintensity were identified: homogeneously hyperintense, hyperintense rim, and cysts with "dot sign." Although "dot sign" cysts were larger $(P<.001)$, there was no significant difference in patient age or the risk of ventricular obstruction among T2 hyperintense cysts. Cyst wall histopathology did not vary with imaging appearance.

CONCLUSIONS: Hyperintensity on T2-FLAIR, whether homogeneous, rim, or "dot sign," is associated with larger cyst size and younger patient age, and is an imaging risk factor for obstructive ventriculomegaly. The hyperintense rim does not represent a thickened cyst wall.

ABBREVIATIONS: $\mathrm{CC}=$ colloid cyst; $C$ CRS = colloid cyst risk score; PAS = periodic acid-Schiff; EMA = epithelial membrane antigen; CDX2 = Caudal Type Homeobox 2; AUC = area under the curve

C olloid cysts (CCs) are benign neoplasms accounting for $0.1 \%$ to $1 \%$ of all intracranial tumors. ${ }^{1}$ Previously thought to be a paraphyseal remnant, ${ }^{2,3}$ more recent histochemical and ultrastructural analyses support an endodermal origin. ${ }^{4-7}$ Although congenital lesions, CCs rarely present in the pediatric population and typically come to attention in the 4 th and 5 th decades. ${ }^{8} \mathrm{CCs}$ occur almost exclusively within the third ventricle near the foramen of Monro where they may obstruct CSF flow resulting in sudden neurologic deterioration and death. ${ }^{9}$ A CC risk score (CCRS) has been proposed in the neurosurgical literature that, in addition to patient age $<65$ years and presence of a headache,

Received March 4, 2020; accepted after revision June 16.

From the Departments of Diagnostic and Interventional Imaging (S.D.K., R.F.R., K.D.W.), Neurosurgery (A.L.D.), and Pathology (M.B.B.), McGovern Medical School, The University of Texas Health Science Center at Houston, Houston, Texas; Department of Quality and Outcomes Management (J.P.F.), Texas Children's Hospital, Houston, Texas.

Previously presented, in part, at: Annual Meeting of the American Society of Neuroradiology, June 2-7, 2018, Vancouver, British Columbia, Canada. incorporates the following imaging variables: risk zone (location in the third ventricle near the foramen of Monro or aqueduct of Sylvius), cyst size $\geq 7 \mathrm{~mm}$, and hyperintensity on T2-FLAIR imaging. ${ }^{10}$ This paper further expands on the imaging appearance of CCs on T2-FLAIR and correlates it with histopathology, cyst size, patient age, and the occurrence of obstructive ventriculomegaly.

\section{MATERIALS AND METHODS}

The study is institutional review board approved and compliant with the Health Insurance Portability and Accountability Act guidelines. This is a retrospective study of 64 patients identified by a tertiary hospital imaging report data base search (Primordial) for "colloid cyst," limited to imaging of the brain. Patients who had a

Please address correspondence to Roy F. Riascos, MD, Professor and Chief of Neuroradiology, Department of Diagnostic and Interventional Imaging, The University of Texas Health Science Center at Houston, Suite MSB 2.130B, 6431 Fannin St, Houston, TX 77030; e-mail: Roy.F.Riascos@uth.tmc.edu

http://dx.doi.org/10.3174/ajnr.A6722 


\begin{tabular}{|c|c|c|c|c|c|}
\hline \multirow[b]{2}{*}{ Signal Intensity on T2-FLAIR } & \multicolumn{3}{|c|}{ Hyperintense $(n=34)$} & \multirow{2}{*}{$\begin{array}{l}\text { Hypointense } \\
\qquad(n=30)\end{array}$} & \multirow[b]{2}{*}{ Total } \\
\hline & Homogeneously Hyperintense & "Dot Sign" & Hyperintense Rim & & \\
\hline Number of patients & 12 & 6 & 16 & 30 & 64 \\
\hline Sex (Male) & $6(50 \%)$ & $3(50 \%)$ & $9(56.2 \%)$ & $13(43.3 \%)$ & 31 (48\%) \\
\hline \multicolumn{6}{|l|}{ Patient age (years) } \\
\hline Mean $(+/-$ SD) & $37.0(23.4 \%)$ & $37.5(15.6 \%)$ & $52.1(22 \%)$ & $55.2(14.7 \%)$ & $49.8(19.8 \%)$ \\
\hline Median & 25.5 & 31.5 & 49.5 & 53 & 50 \\
\hline \multicolumn{6}{|l|}{ Cyst size (mm) } \\
\hline Mean & 10.6 & 16.8 & 11.1 & 6.8 & 9.6 \\
\hline Median & 11.0 & 15.0 & 12.5 & 6.3 & 9.0 \\
\hline Obstructive ventriculomegaly & $8(67 \%)$ & $5(83.3 \%)$ & $8(50 \%)$ & $3(10 \%)$ & $24(37.5 \%)$ \\
\hline $\begin{array}{l}\mathrm{CT}^{\mathrm{a}} \text { (hyperattenuated/ } \\
\text { iso-hypoattenuated) }\end{array}$ & $4 / 4$ & $0 / 5$ & $14 / 0$ & $20 / 0$ & $38 / 9$ \\
\hline $\begin{array}{l}\text { T1 signal (hyper-/iso-/ } \\
\text { hypointense) }\end{array}$ & $1 / 5 / 6$ & $5 / 1 / 0$ & $10 / 5 / 1$ & $16 / 14 / 0$ & $32 / 25 / 7$ \\
\hline Zone II & 3 & 0 & 2 & 6 & 11 \\
\hline $\begin{array}{l}\text { Histopathology available } \\
\text { for review }\end{array}$ & 8 & 3 & 10 & 7 & 28 \\
\hline
\end{tabular}

missing sequence (T1, T2, or T2-FLAIR), did not have a confirmed diagnosis of CC on review of the MR imaging, or had significant artifacts that precluded evaluation were excluded. Patients with cysts measuring less than $4 \mathrm{~mm}$ were excluded as characterization in these cases was difficult. Noncontrast MR imaging was available for all 64 patients with postcontrast T1WI in 38 patients. CT images were available for 47 patients.

\section{Imaging Evaluation}

MR imaging scans were performed on 1.5 and 3T MR imaging machines (Ingenia 3T, Achieva 3T, Intera 1.5T, Philips Healthcare; HDXT 3T and Excite 1.5T, GE Healthcare). For this retrospective study, no specific imaging protocol was used. All noncontrast MRI of the brain had axial T1, T2, and T2-FLAIR, sagittal T1, DWI, and axial T2* (GRE or SWI) sequences. Postcontrast, T1 fat-suppressed imaging was performed in 3 orthogonal planes after administration of MultiHance ( $0.1 \mathrm{mmol} / \mathrm{kg}$ body weight; Bracco Diagnostics). All CT scans were performed as routine brain on 64- and 128-section scanners with 3 orthogonal planes available for interpretation.

The imaging was reviewed by a senior neuroradiologist ( 27 years of experience) and a neuroradiology fellow. The following imaging features were recorded: cyst size (greatest axial dimension), risk zone location as described by Beaumont et al, ${ }^{10}$ enhancement pattern, diffusion restriction, calcification, hemorrhage, and presence or absence of a hyperintense rim, "black hole," or an intracystic nodule ("dot sign"). ${ }^{10-12}$ Signal intensity on T1, T2, and T2-FLAIR imaging was recorded relative to that of adjacent gray matter. Obstructive ventriculomegaly was recorded if there was disproportionate dilation of the lateral ventricles relative to the sulci without enlargement of the third ventricle. Attenuation on CT was recorded relative to adjacent gray matter.

Based on the T2-FLAIR sequence, the CCs were divided into 2 groups: hyperintense and hypointense cysts. Hyperintense cysts were further subdivided into 3 categories: 1) homogeneously hyperintense; 2) hyperintense with a "dot sign"; 3) thin hyperintense rim with a much larger iso- to hypointense core. These groups were correlated with patient age, cyst size, and the presence of obstructive ventriculomegaly. Blinded review of the pathology was undertaken by a senior neuropathologist with 35 years of experience. Histopathology was correlated with the imaging appearance of the cyst.

\section{Statistical Methods}

The independent $t$ test was used to compare differences in median age and cyst size for the overall hyperintense and hypointense cohorts. Nonparametric Wilcoxon rank-sum and Fisher exact tests were then used to conduct a further subgroup analysis of continuous variables (ie, age and cyst size) and the presence of obstructive ventriculomegaly, respectively. The distribution and normality were assessed by using standard graphic methods and kurtosis values. Level of significance was set at $P<.05$. Statistical analysis was performed by using STATA for Windows (version 15.0, StataCorp).

\section{Pathologic Correlation}

Histopathology was available for review in 28 cases. Cysts were examined by using H\&E, supplemented with special stains: periodic acid-Schiff (PAS), mucicarmine, and Masson trichrome stains, and immunohistochemistry analysis with antibodies for Pan-keratin, CAM 5.2, CK-7, Keratin 20, epithelial membrane antigen (EMA), Caudal Type Homeobox 2 (CDX2), and glial fibrillary acidic protein. Not all cysts were examined as specimens sometimes contained cyst contents only, without the cyst wall.

\section{RESULTS}

The 64-patient cohort had an median age of 50 years (range $=10$ to 99 years) and $52 \%$ were female. Median cyst size was $9.0 \mathrm{~mm}$. Most of cysts were located in risk zone I, 17.1\% were in zone II, and none were in zone III as defined by Beaumont et al. ${ }^{10}$ Obstructive ventriculomegaly was present in 37.5\% (24/64) of patients. All cysts with associated obstructive ventriculomegaly were in zone I (Table). 


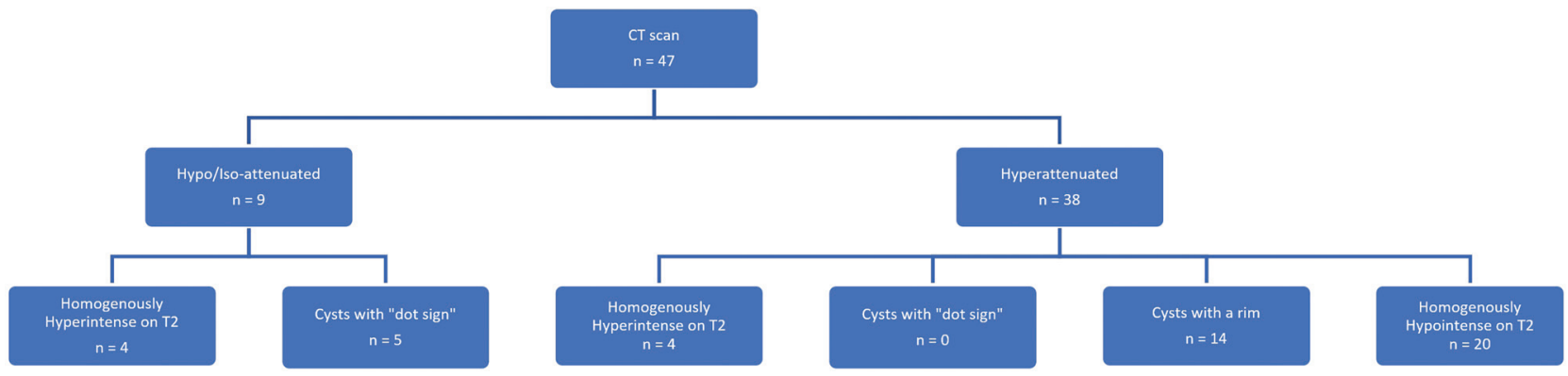

FIG 1. An overview of the imaging appearance of third ventricular colloid cyst on CT and corresponding MR imaging characteristics.

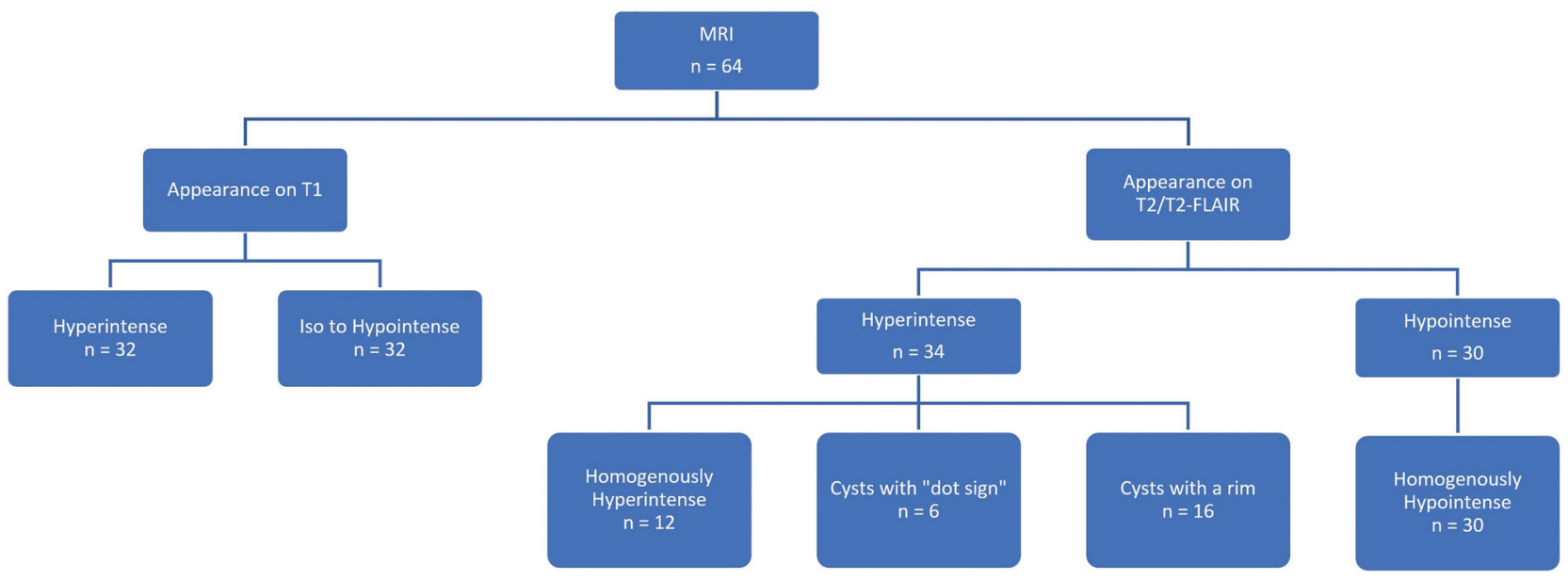

FIG 2. An overview of the imaging appearance of third ventricular colloid cyst on MR imaging.

\section{CT}

CT was available for 47 patients (Fig 1). Most colloid cysts (81\%) were hyperattenuated with 7 iso-attenuated and 2 hypoattenuated relative to adjacent gray matter. In no case was a cyst iso-attenuated with CSF. Calcification was not identified within any cyst or cyst wall.

\section{MRI}

All 64 patients had an MR imaging, with postcontrast imaging available for 38 patients. Half of the cysts were hyperintense on the T1WI. No cyst was isointense to CSF on the T1WI. Although some cysts showed minimal, discontinuous enhancement along the wall it was likely due to adjacent vessels rather than true enhancement, no thick rimlike or nodular enhancement was seen. None of the cysts showed evidence of restricted diffusion, nor was there susceptibility artifact suggesting recent or remote hemorrhage.

Based on the appearance on T2-FLAIR, CCs were divided into hyperintense (34 patients) and hypointense groups (30 patients) (Fig 2). Median age was significantly lower (43.5 versus 53 years, $P=.01$ ), median cyst size was larger (12 versus $6.3 \mathrm{~mm}, P<.0001)$, and the risk of obstructive ventriculomegaly was higher (relative risk $6.18,95 \%$ CI $[2.04,18.67]$ ) in the hyperintense group. Hyperintensity on T2-FLAIR appeared in 3 different patterns: homogeneously hyperintense, cysts with rim, and cysts with "dot sign." Homogeneously hypointense cysts had no internal variability in signal. Relationship of demographics, obstructive ventricfulomegaly, and appearance of colloid cysts on CT and MR imaging is summarized in the Table.

\section{Homogeneously Hypointense Cysts $(n=30)$}

These cysts were uniformly hypointense on T2 and T2-FLAIR sequences and constituted the most common appearance in our study. These cysts did not have an apparent rim or varying signal intensity core. There was no evidence of susceptibility artifact to account for the low signal. All cysts in this category were uniformly hyperattenuated and well-circumscribed on CT. The median age at presentation in this group of patients was 53 years with a median cyst diameter of $6.3 \mathrm{~mm}$ (range: $4 \mathrm{~mm}$ to $12 \mathrm{~mm}$ ). Only $10 \%$ of these patients presented with ventriculomegaly $(P<.001)$.

\section{Homogeneously Hyperintense Cysts ( $n=12)$}

These cysts were uniformly hyperintense on T2 and T2-FLAIR sequences (Fig 3). They were associated with a significantly higher risk of obstructive ventriculomegaly (67\% patients, $P<.001)$ in comparison to homogeneously hypointense cysts, but had a similar risk in comparison with other hyperintense cysts. The median cyst size in this group was significantly higher than homogeneously hypointense cysts (11 versus $6.3 \mathrm{~mm}$, $P<.001)$. Median age of presentation was lower than in patients with homogeneously hypointense cysts ( 25.5 years versus 53 years, 


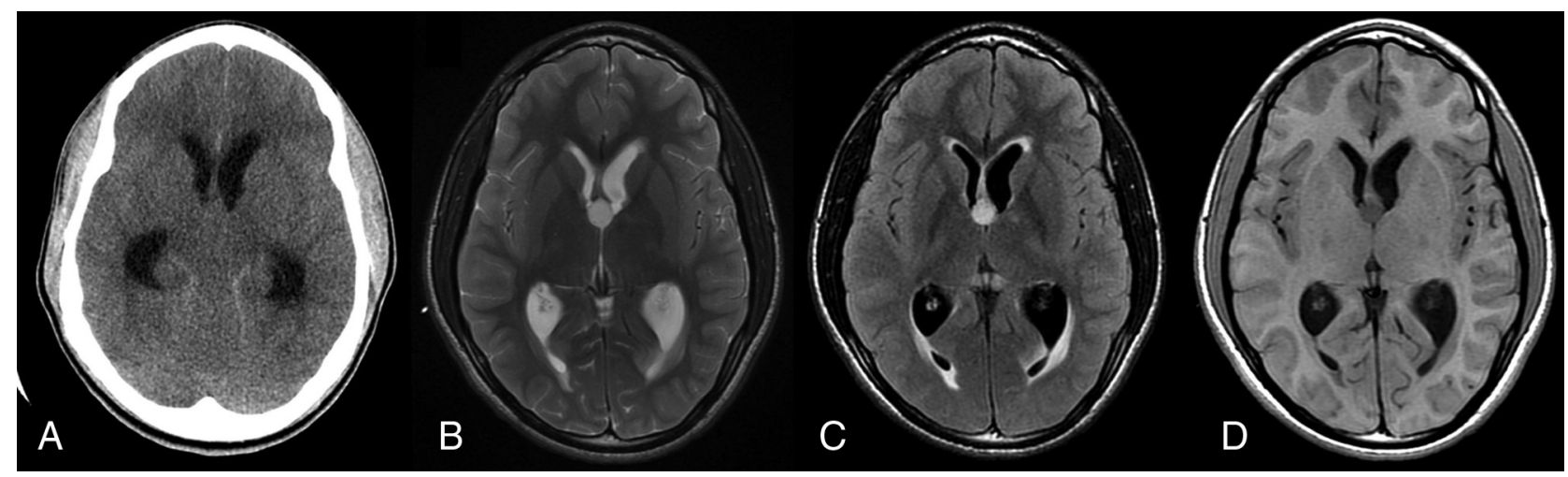

FIG 3. Homogeneously hyperintense cyst: 13-year-old boy found unconscious after complaining of headache for 2 days. Initial CT demonstrates dilation of the lateral ventricles. However, the colloid cyst was difficult to detect as it was isointense relative to the adjacent brain. Subsequent MR imaging showed a colloid cyst homogeneously hyperintense on T2 (B) and T2-FLAIR weighted images (C) and hypointense on T1-weighted axial image $(D)$. Also, note the presence of periventricular edema associated with ventriculomegaly.

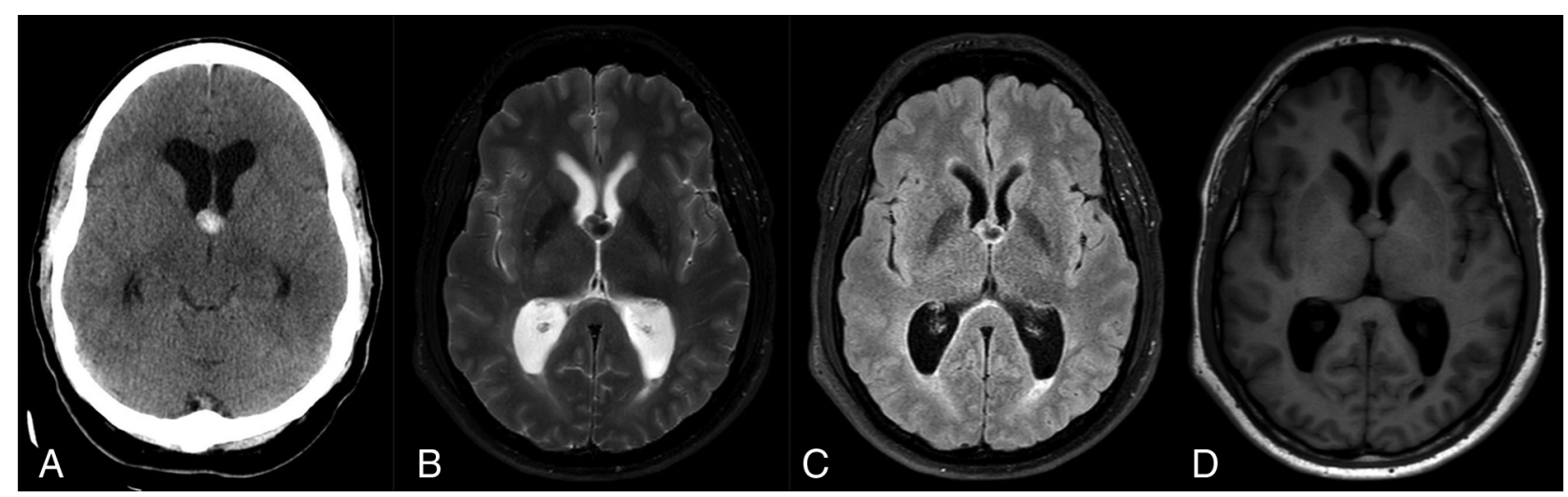

FIG 4. Cyst with rim: 55-year-old woman with a 6-week history of headache. Non-contrast CT (A) shows a hyperattenuated colloid cyst measuring $13 \mathrm{~mm}$ at the roof of third ventricle. On T2WI (B), the colloid cyst has a hypointense core with a thin hyperintense rim, which is more pronounced on T2-FLAIR (C). The cyst is mildly heterogeneous and isointense centrally on T1-weighted image (D). There was no evidence of hemorrhage on the GRE sequence (image not shown). There is associated enlargement of the lateral ventricles with periventricular edema.

$P=.01)$. Half of the cysts were uniformly hypoattenuated and half uniformly hyperattenuated on CT.

\section{Cysts with "Dot Sign" (n=6)}

These cysts were almost entirely hyperintense on T2WI and T2FLAIR with a tiny, $<5 \mathrm{~mm}$, internal hypointense nodule that has been previously described in the imaging literature as the "dot sign." These "dots" were significantly smaller than the hypointense "cores" described in the "cysts with rim." Five out of 6 cysts with the dot sign were hyperintense on the T1WI; therefore, the nodule was not visualized on T1WI. Obstructive ventriculomegaly was significantly more common than in homogeneously hypointense cysts (83.3\% versus $10 \%, P<.001)$. These cysts were significantly larger than both homogeneously hypointense cysts (median diameter of $15 \mathrm{~mm}$ versus $6.3 \mathrm{~mm}, P<.001)$ and other hyperintense cysts $(P=.007$ and $P=.03$ for homogeneously hyperintense and cysts with rim, respectively). The median age at presentation was significantly lower than in patients with homogeneously hypointense cysts ( 31.5 versus 53 years, $P<.001$ ) but similar to those with other hyperintense cysts. These cysts were iso- to hypoattenuated on CT with a punctate area of hyperattenuation corresponding to the intracystic nodule identified in 1 case.

\section{Cysts with $\operatorname{Rim}(n=16)$}

These cysts had a hyperintense rim and an iso- to hypointense central core that occupied at least $50 \%$ of the total cyst on T2WI and T2-FLAIR (Fig 4). Cysts with markedly hypointense cores have been previously termed as having a "black hole" appearance. Correlating the MR imaging appearance with CT revealed that the hypointense cores were hyperattenuated on CT (Fig 1). Cysts with hyperintense rims were larger than the homogeneously hypointense cysts (median diameter of 12.5 versus $6.3, P<.001$ ). Obstructive ventriculomegaly occurred in $50 \%$ of these patients which was significantly higher than in the homogeneously hypointense group $(P=.02)$, but similar to those with other hyperintense cysts.

\section{Histopathology}

The contents of colloid cysts, available for histopathologic examination in 28 cases, consisted of amorphous, proteinaceous, and acellular gelatinous material. Histopathology was available for $3 / 6$ cysts with "dot sign" on imaging. The hypointense "dot" corresponded to an intracystic nodule in 1 case and was described grossly as a gelatinous papillary protrusion. The nodule was not 


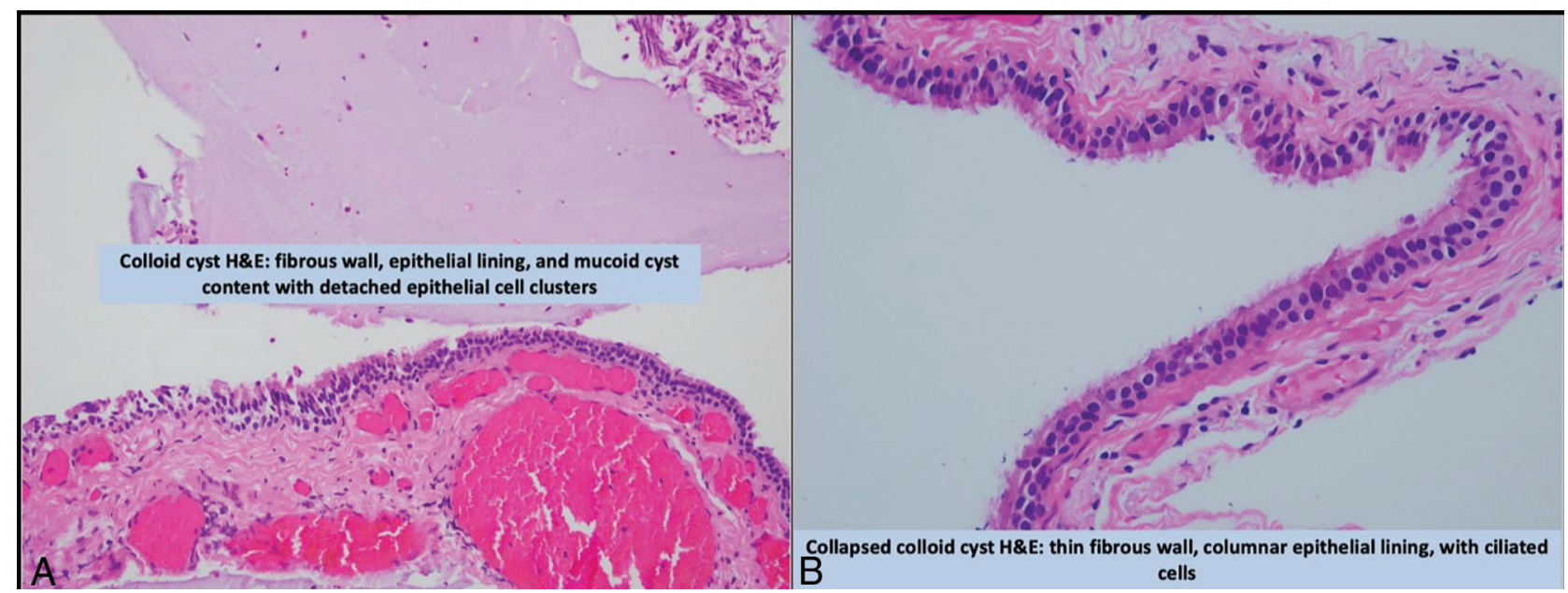

FIG 5. Hematoxylin and Eosin staining of a typical homogeneously hyperintense cyst on T2 and T2-FLAIR images. The cyst wall is composed of columnar, ciliated epithelial cells. The wall was not thickened and there was not an abnormal increase in goblet cells or any cellular atypia. Contents of the cysts consisted of a mucoid material with detached epithelial clusters of cells.

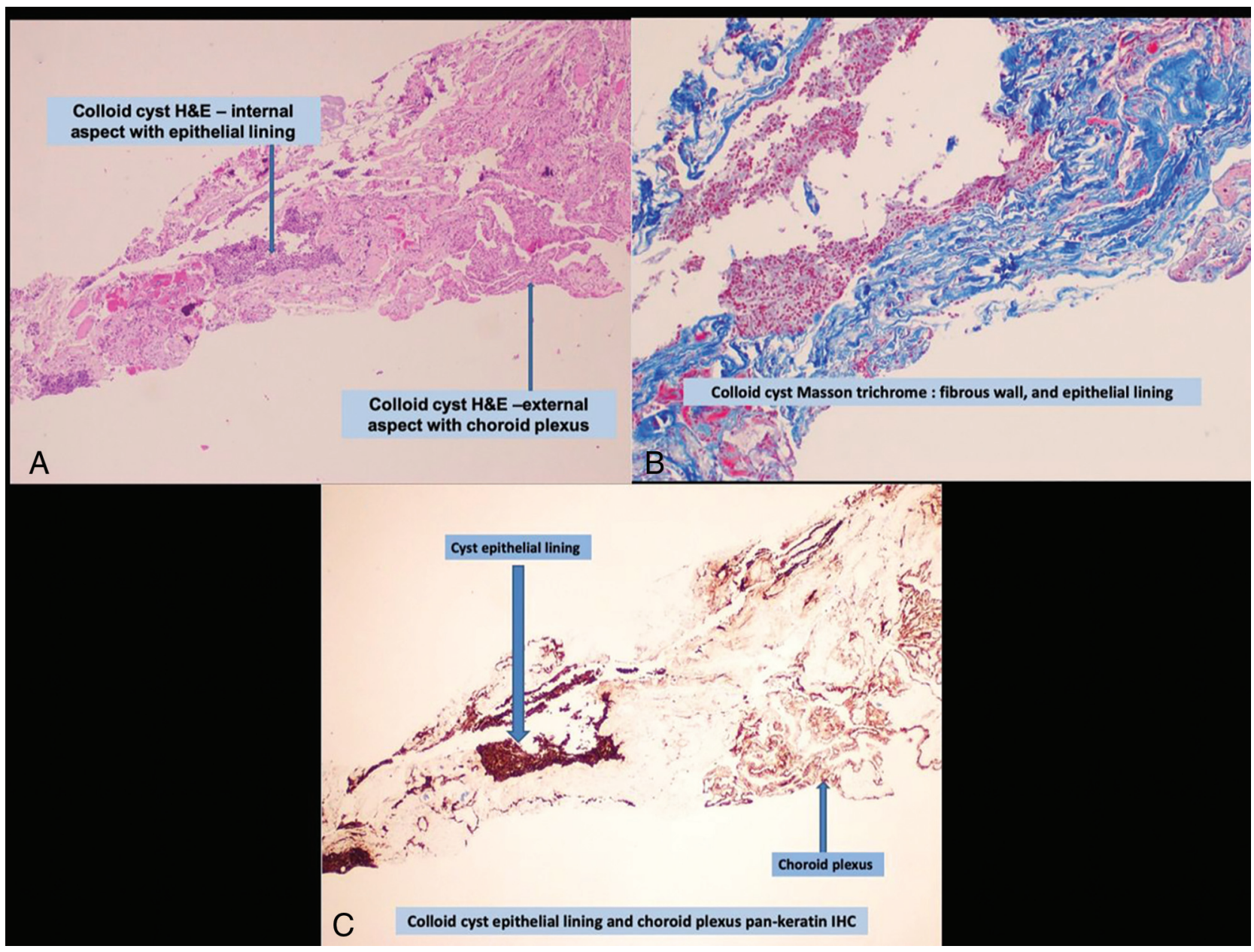

FIG 6. Pathologic examination of a cyst that had the "cysts with rim" appearance revealed a viscous plug of colloid material which was PAS-positive. The cyst wall consisted of a thin fibrous capsule and a lining of partly ciliated cuboidal to columnar epithelium (A). The fibrous wall, and the associated choroid plexus stroma are highlighted by the Masson trichrome stain (B). The cyst epithelium is positive for CAM 5.2, pankeratin (C), EMA, and CK7; it is negative for CDX2. The peripheral cyst wall was not unusually thickened and was approximately 1-3 microns thick, and therefore would not have been visible on MR imaging. 
separately described in the examination of the other 2 cysts that were received in fragments.

Despite the varied MR imaging appearance on histopathological examination, the appearance of colloid cysts was monotonous with a very thin fibrous wall lined by a single to pseudostratified layer of columnar-cuboidal, partially ciliated epithelium with goblet cells infrequently seen (Figs 5 and 6). The cyst wall was not significantly thicker in cysts with rim. Choroid plexus was identified in 4 specimens. Calcification was present in 1 cyst wall that was not visible, even retrospectively, on GRE-T2*WI. There was evidence of prior hemorrhage in the wall of 1 "dot sign" cyst that was not visible on imaging.

\section{DISCUSSION}

Although increasingly diagnosed as an incidental finding, third ventricular colloid cysts are well known for their potential to become symptomatic by creating ventricular obstruction often with serious outcome. ${ }^{13,14}$ Various clinical and imaging characteristics have been studied in hopes of determining which cysts are most likely to become symptomatic. Prior neurosurgical literature has noted an increased incidence of symptoms and rapid deterioration in pediatric patients with "watery cysts," hyperintense on T2-weighted and T2-FLAIR imaging. ${ }^{15,16}$

A relationship between cyst signal intensity and symptomatology was first demonstrated in the adult population by Pollock et $\mathrm{al}^{17}$ who observed that hyperintensity on T2WI, in addition to cyst size, patient age, ventriculomegaly, and headache, was significantly associated with symptomatic cysts. Although MR imaging was available for only one-third of patients and there was no comment regarding cyst appearance on T2-FLAIR, they found a 5 -fold increase in hyperintense signal on T2WI in patients who were symptomatic. Interestingly, $71 \%$ of their hyperintense cysts were large, measuring $\geq 10 \mathrm{~mm}^{17}$

Variables thought to be predictive of symptomatic status were further explored by Beaumont et al, ${ }^{10}$ who combined demographic, clinical, and imaging factors to form the CCRS which is a system for use by neurosurgeons to attempt to identify patients at highest risk of having a symptomatic cyst and to stratify the risk of hydrocephalus. In the CCRS, a point is awarded for: age $<65$; cyst diameter $\geq 7 \mathrm{~mm}$; presence of a headache at presentation; risk zone location; and the presence of hyperintensity on T2-FLAIR. On a 0 - to 5-point scale, a score of $\leq 2$ is considered low risk, whereas a score of $\geq 4$ is associated with a high risk of symptoms and obstructive hydrocephalus. A score of 3 represents an intermediate risk. Cysts with T2-FLAIR hyperintensity were significantly more likely to be symptomatic (relative risk $=2.4$ ) and to present with hydrocephalus (relative risk = 5.1) than those without hyperintensity. Remarkably, they reported that all but 1 cyst with associated obstructive hydrocephalus (96.6\%) were hyperintense on the T2-FLAIR sequence. Although hyperintensity on T2WI was not significant for symptomatic cysts, it was significantly associated, but to a lesser degree than FLAIR, with hydrocephalus. The discrepancy in the recording of a cyst as hyperintense on T2-FLAIR, but not on T2-weighted imaging, may be due to increased conspicuity of this hyperintensity when surrounding CSF is suppressed. This would most likely be a factor in cysts with rim or smaller cysts. It is also possible that T1- shortening may also play a role even though there was no relationship between hyperintensity on the T1-weighted images and cysts being symptomatic in our study and the work of others. ${ }^{10,17}$ Further, in our study, there was no association between cyst hyperintensity on T2-FLAIR and T1-shortening evidenced on the T1WI, likely due to the dominant effect of T2-shortening on T2weighted imaging including the T2-FLAIR sequence. Beaumont et $\mathrm{al}^{10}$ speculated that a "protein doping" effect within the cyst secondary to an osmotic phenomenon was responsible for the hyperintensity on FLAIR that was more conspicuous relative to T2-weighted images.

The CCRS has recently been externally validated as having excellent discriminatory capacity for symptomatic (area under the curve $[$ AUC $]=0.914)$ and obstructive hydrocephalus (AUC = 0.943). In this validation study, cyst appearance was reported only for the T2-FLAIR sequence and not precisely defined, but was available for all 156 patients. ${ }^{18}$ The presence of hyperintensity on T2-FLAIR was significantly associated with symptomatic status $(\mathrm{OR}=6.4)$ and obstructive hydrocephalus ( $\mathrm{OR}=5.72)$. Hyperintensity was identified in $53 \%$ of symptomatic cysts and $56 \%$ of those creating obstructive hydrocephalus but was found in only $18 \%$ of those that were asymptomatic. Cyst size, presence of a headache, and risk zone location were also significant factors.

Our observation that T2-FLAIR signal hyperintensity is associated with ventricular obstruction in patients with CCs supports the findings of these prior studies. We observed that patients with hyperintense cysts were likely to present at a younger age and have larger cysts. A plausible justification for increased risk of ventriculomegaly in hyperintense cysts may to some extent be due to their larger size. A hypothetical explanation for association of T2-FLAIR hyperintensity and cyst symptomatology has been suggested by Beaumont et $\mathrm{al}^{10}$ and Melhem et $\mathrm{al}^{19}$; higher protein concentration within the cyst is associated with hyperintensity on T2-FLAIR sequence. A higher concentration of protein may create an osmotically driven influx of free water resulting in cyst enlargement, subsequent obstruction of the foramen of Monro, and ventriculomegaly. A lowering of protein concentration and increased free water would lead to T2 prolongation. Thus, the cysts that are hyperintense on T2-FLAIR are more likely to enlarge and become symptomatic. We found that hyperintensity on T2-FLAIR manifests in one of three main patterns, homogeneously hyperintense, hyperintense rim, and "dot sign;" all of which are associated with larger cyst size, younger patient age, and a higher risk of obstructive ventriculomegaly in comparison to homogeneously hypointense cysts. Of the hyperintense cysts, those with the "dot sign" were significantly larger but were otherwise similar to other hyperintense cysts.

The histopathologic appearance of the cyst wall was monotonous and did not substantially vary despite significant differences in the appearance of the cysts on imaging (Figs 5 and 6). We postulate that the hyperintense rim represents a peripheral hydration layer, which accounts for the increase in T2 and T2-FLAIR signal around a hypointense, viscous central core. The CT appearance of cysts with rim is also in agreement as, on close examination, the hyperattenuated central core is surrounded by a subtle lower attenuation periphery, with an ill-defined border between the regions of differing attenuation. In prior radiologic-pathologic 
correlation reports regarding the "black hole" appearance, the center has been found to be highly viscous (like "motor oil") or even described as solid, whereas the high signal rim has been described as liquid and consisting of amorphous material with cellular debris, erythrocytes, and cholesterol clefts. ${ }^{20,21}$ Lack of susceptibility artifact on the SWI and diffusion-weighted imaging, within the center of the black hole cysts supports prior investigations, which have stated that neither paramagnetic metals nor hemorrhage account for the T2-shortening seen within the black hole cysts. ${ }^{11}$

In keeping with the literature, most of our cysts were hyperattenuated on CT. ${ }^{10,16,17}$ In general, higher attenuation on CT corresponded to lower signal on the T2WI; both believed to indicate higher viscosity which correlates with difficult aspiration in the surgical literature. ${ }^{5,22}$

Half of the cysts were hyperintense on the T1-weighted images. This T1-shortening, as reported in prior literature, is likely related to higher protein and cholesterol content and is not due to calcium, metal, or blood products. ${ }^{11,23}$ Interestingly, cysts with the "dot sign" were high in signal intensity not only on both T2 and T2-FLAIR, but also on T1-weighted imaging indicating a higher protein and/or cholesterol content. In vitro studies of protein solutions have shown that $\mathrm{T} 1$ hyperintensity is seen with protein concentrations exceeding $9 \mathrm{~g} / \mathrm{dL}{ }^{24}$ At extremely high concentrations, greater than $17 \mathrm{~g} / \mathrm{dL}$, in vitro solutions of protein remained high in signal on the T1-weighted images but T2-shortening effects predominated so that the solutions became dark on T2WI. We did not observe any association between the $\mathrm{CT}$ attenuation or T1 hyperintensity with symptomatic clinical presentation, which is in keeping with the existing imaging literature. ${ }^{10,17}$

Calcification has rarely been reported in the walls of colloid cysts, but was not seen in any of our cases. ${ }^{25}$ On histopathologic examination, our specimens often contained choroid plexus, as it is closely juxtaposed to the cyst wall. In some of these cases, it seems likely that calcification in psammoma bodies, which are intrinsic to the choroid plexus, may be mistakenly attributed to the colloid cyst wall (Fig 6). Although we did not discover any element of hemorrhage within our colloid cysts, hemorrhagic cysts are well reported in the literature and may be a cause of abrupt neurologic decline. ${ }^{26}$

The limitations of our study include the retrospective design and relatively small sample size in the subgrouping of hyperintense cysts. The lack of a uniform imaging protocol due to retrospective nature of the study is another limitation. Pathologic examination was often limited because the specimen sometimes lacked the cyst wall. We used obstructive ventriculomegaly as an "end point" and did not address the degree of ventriculomegaly due to the complex interdependence of many variables including ventricular compliance, intermittent obstruction scenarios, lack of recognition of subtle, or early, ventricular dilation as well as the marked variation in the time from symptom onset to imaging.

\section{CONCLUSIONS}

Three different imaging subtypes of T2-FLAIR hyperintense cysts are observed: homogeneously hyperintense; cysts with rim; and cysts with "dot sign." These T2-FLAIR hyperintense CCs are associated with younger patient age, larger cyst size, and a greater occurrence of obstructive ventriculomegaly than cysts with no hyperintensity. No association was observed between CT attenuation or T1 hyperintensity and obstructive ventriculomegaly. The hyperintense rim of a "black hole" cyst does not represent a thickened cyst wall.

\section{REFERENCES}

1. McLendon RE, Rosenblum MK, Bigner DD. Russell \& Rubinstein's Pathology of Tumors of the Nervous System 3rd ed. CRC Press; 2006

2. Kappers JA. The development of the paraphysis cerebri in man with comments on its relationship to the intercolumnar tubercle and its significance for the origin of cystic tumors in the third ventricle. $J$ Comp Neurol 1955;102:425-509 CrossRef Medline

3. Shuangshoti S, Roberts MP, Netsky MG. Neuroepithelial (colloid) cysts: pathogenesis and relation to choroid plexus and ependyma. Arch Pathol 1965;80:214-24 Medline

4. Tsuchida T, Hruban RH, Carson BS, et al. Colloid cysts of the third ventricle: immunohistochemical evidence for nonneuroepithelial differentiation. Hum Pathol 1992;23:811-16 CrossRef Medline

5. Kondziolka D, Lunsford LD. Stereotactic management of colloid cysts: factors predicting success. J Neurosurg 1991;75:45-51 CrossRef Medline

6. Ho KL, Garcia JH. Colloid cysts of the third ventricle: ultrastructural features are compatible with endodermal derivation. Acta Neuropathol 1992;83:605-12 CrossRef Medline

7. Graziani N, Dufour H, Figarella-Branger D, et al. Do the suprasellar neurenteric cyst, the Rathke cleft cyst and the colloid cyst constitute a same entity? Acta Neurochir (Wien) 1995;133:174-80 CrossRef Medline

8. Macdonald RL, Humphreys RP, Rutka JT, et al. Colloid cysts in children. Pediatr Neurosurg 1994;20:169-77 CrossRef Medline

9. de Witt Hamer PC, Verstegen MJT, De Haan RJ, et al. High risk of acute deterioration in patients harboring symptomatic colloid cysts of the third ventricle. J Neurosurg 2002;96:1041-45 CrossRef Medline

10. Beaumont TL, Limbrick DD, Rich KM, et al. Natural history of colloid cysts of the third ventricle. JNS 2016;125:1420-30 CrossRef Medline

11. Maeder PP, Holtås SL, Basibüyük LN, et al. Colloid cysts of the third ventricle: correlation of MR and CT findings with histology and chemical analysis. AJR Am J Roentgenol 1990;155:135-41 CrossRef Medline

12. Osborn AG, Hedlund GL, Salzman KL. Introduction to neoplasms, cysts and tumor-like lesions. Osborne's Brain, 2nd ed. 2018 Elsevier Health Sciences

13. Little JR, MacCarty CS. Colloid cysts of the third ventricle. J Neurosurg 1974;40:230-35 CrossRef Medline

14. Mathiesen T, Grane P, Lindgren L, et al. Third ventricle colloid cysts: a consecutive 12-year series. J Neurosurg 1997;86:5-12 CrossRef Medline

15. Kumar V, Behari S, Kumar Singh R, et al. Pediatric colloid cysts of the third ventricle: management considerations. Acta Neurochir (Wien) 2010;152:451-61 CrossRef Medline

16. Alnaghmoosh N, Alkhani A. Colloid cysts in children, a clinical and radiological study. Childs Nerv Syst 2006;22:514-16 CrossRef Medline

17. Pollock BE, Schreiner SA, Huston J. A theory on the natural history of colloid cysts of the third ventricle. Neurosurgery 2000;46:1077-83 CrossRef Medline

18. Alford EN, Rotman LE, Shank CD, et al. Independent validation of the colloid cyst risk score to predict symptoms and hydrocephalus in patients with colloid cysts of the third ventricle. World Neurosurg 2020;134:e747-53 CrossRef Medline

19. Melhem ER, Jara H, Eustace S. Fluid-attenuated inversion recovery MR imaging: identification of protein concentration thresholds for 
CSF hyperintensity. AJR Am J Roentgenol 1997;169:859-62 CrossRef Medline

20. Urso JA, Ross GJ, Parker RK, et al. Colloid cyst of the third ventricle: radiologic-pathologic correlation. J Comput Assist Tomogr 1998;22:52427 CrossRef Medline

21. Wilms V, Marchal L, Hecke L, et al. Colloid cysts of the third ventricle: MR findings. J Comput Assist Tomogr 1990;14:527-31 CrossRef Medline

22. Khoury CE, Brugières $P$, Decq P, et al. Colloid cysts of the third ventricle: are MR imaging patterns predictive of difficulty with percutaneous treatment? AJNR Am J Neuroradiol 2000;21:489-92 Medline
23. Armao D, Castillo M, Chen $\mathrm{H}$, et al. Colloid cyst of the third ventricle: imaging-pathologic correlation. AJNR Am J Neuroradiol 2000;21:1470-77 Medline

24. Ahmadi J, Destian S, Apuzzo ML, et al. Cystic fluid in craniopharyngiomas: MR imaging and quantitative analysis. Radiology 1992;182:78385 CrossRef Medline

25. Türe U, Hiçdönmez T, Elmaci I, et al. Solid-calcified colloid cyst of the third ventricle. Clin Neurol Neurosurg 2001;103:51-55 CrossRef Medline

26. Beems T, Menovsky T, Lammens M. Hemorrhagic colloid cyst. Surg Neurol 2006;65:84-86 CrossRef Medline 